\title{
Development of advanced hybrid materials with help of pulse- electrodeposition
}

\author{
Uta Klement*, Nooshin Mortazavi, Antonio Mulone, Daniel Melciu and Navin Maidee
}

Materials and Manufacturing Technology, Chalmers University of Technology, Rännvägen 2A, 41296 Gothenburg, Sweden

*uta.klement@chalmers.se, Phone: +46 317721264

\begin{abstract}
Pulse-electrodeposition has been applied to enhance properties of two different types of lightweight construction materials, a periodic cellular material (PCM) and a micro-sandwich. For the PCM, the deformation behaviour of the nanocrystalline Ni-18wt.\%Fe sleeve material (bulk samples) has been investigated up to $548 \mathrm{~K}$. The material exhibits plasticity of $>30 \%$ fracture strain at higher temperatures compared to $<15 \%$ at room temperature. TEM characterization shows that coarser grains are present which enable strain hardening by intragranular dislocation accumulation. This leads to larger fracture strains at higher temperatures. Hence, for allowing application of the PCM at elevated temperatures, the sleeve material has to be stabilized against deformation-induced grain growth. For the micro-sandwich, the pulse-electrodeposited Nickel coating on the face sheets or polymer fibres of the sandwich core can provide extra strength. With respect to the fibres, the plating process needs to be improved further to achieve a continuous and homogeneous coating.
\end{abstract}

Keywords: Electrodeposition, nanocrystalline coating, periodic cellular materials, microsandwich, strength increase, thermal stability, TEM, EBSD

\section{Introduction}

Sandwich structures are widely used in modern lightweight construction e.g. in a variety of transport industries (aerospace, marine, and automobile sector) and in components where properties like low weight, formability and/or damping are required. These structures are layered composites designed to replace their monolithic counterparts in order to reduce weight and material cost. They usually consist of a relatively thick, lightweight core material which is covered by thin and strong face sheets. The core material is often a low-strength material such as a honeycomb, metallic foam or polymer foam. Metallic foams are an attractive option for weight-limited engineering applications [1]. Low relative densities (e.g. a few percent of the parent metal) are achieved through an internal void structure which can be either connected (open-cell) or unconnected (closed-cell) [2]. Bouwhuis and Hibbard [3] generated nanocrystalline hybrid metallic foams by reinforcing conventional open-cell aluminium foams by electrodeposition of a high-strength nanocrystalline sleeve. While stronger than conventional metallic foams, the approach had practical limitations. In particular, the complex and irregular architecture of the foam made it difficult to obtain uniform electrodeposition over the internal ligaments. The inner ligaments had thinner electrodeposited sleeves which as a consequence collapsed first during mechanical testing. Evidently, this limited the achievable strength increase. However, the electrodeposition uniformity issue could be eliminated by making use of periodic cellular materials (PCMs). In PCMs, the total material mass is reduced by retaining only that part of the material which has geometrically-high load-bearing efficiency. Hence, PCMs are more structurally efficient than conventional stochastic foams [4,5]. A range of investigations was performed by applying 
nanocrystalline sleeves on both polymeric and metallic PCMs [3, 6-10]. In this paper, challenges with the pulse electrodeposited nanocrystalline coating used for property enhancement of advanced hybrid materials in a periodic cellular material (PCM) and a microsandwich are discussed. Special emphasis is laid on the microstructural characterization of the electrodeposited material.

\section{Experimental}

For producing the nanocrystalline hybrid metallic material, the periodic cellular pre-form was produced by stretch-forming of a perforated copper sheet. Afterwards, a Ni-18wt.\%Fe nanocrystalline sleeve was pulse-electrodeposited around the pre-form at Integran Technologies Inc., Toronto, Canada. Moreover, also a 5 mm thick plate of nanocrystalline Ni-18wt.\%Fe was produced. Using wire electrical discharge machining, cylindrical samples of $\sim 2 \mathrm{~mm}$ in diameter and $\sim 4 \mathrm{~mm}$ in length were cut from the plate. Both the cylindrical samples and the $\mathrm{Cu} /$ nano-Ni-Fe PCM were tested at a strain rate of $10^{-4} \mathrm{~s}^{-1}$ in an Instron 8032 tensile test machine equipped with a thermal chamber. Prior to the compression tests, the samples were annealed for 30 minutes at the respective temperature. The Hybrix ${ }^{\mathrm{TM}}$ microsandwich was provided by Lamera AB, Sweden, and consisted of stainless steel sheets to which polyamide fibres were attached by an epoxy. For pulse-electrodeposition of a nanocrystalline coating, a Nickel sulfamate bath and different plating conditions were used.

Investigations by use of electron backscatter diffraction (EBSD) technique were performed in a LEO 1550 field emission gun scanning electron microscope which was equipped with HKL Channel 5 EBSD system and Nordlys II detector. To obtain orientation maps, an accelerating voltage of $10 \mathrm{kV}$ and a step size of 10,20 , and $100 \mathrm{~nm}$ was used, respectively. Samples were investigated in the ordinary EBSD setup ( $70^{\circ}$ tilt of sample towards the EBSD detector) and in transmission-EBSD setup [11] using samples prepared for transmission electron microscopy (TEM). For microstructural investigations, a Zeiss 912 Omega transmission electron microscope, operating at an accelerating voltage of $120 \mathrm{kV}$, was used. TEM samples were prepared by usual dimple grinding and ion polishing. EBSD samples were prepared by electropolishing (10 s at a voltage of $31 \mathrm{~V}$ ) using Struers A2 electrolyte in a Struers LectroPol-5 equipment. Nanoindentation was performed at 60 measuring points each using a force of $1 \mathrm{mN}$ and $5 \mathrm{mN}$.

\section{Results}

\subsection{Nanocrystalline hybrid PCM}

Figure 1(a) shows a typical PCM structure that consists of a Cu core and a nanocrystalline NiFe coating. The nanocrystalline coating is about $100 \mathrm{~nm}$ thick and has an average grain size of $31 \pm 12 \mathrm{~nm}$ [12]. During the structural collapse of such nanocrystalline hybrid PCMs, different failure mechanisms are observed $[9,13,14]$. While in a constrained compression setup the confined nodes induce axial compressive stresses into the struts which lead to strut failure by buckling [9], the uniaxial free compression induces transverse bending stresses which cause stress concentrations at the nodes of the struts where cracks are formed (Fig. 1(b)). Also plastic wrinkling and cracks at the hinges of the PCM can be observed. Details about the compression test including the stress-strain curve are provided in $[14,15]$. Since the dominant failure mechanisms are in the nanocrystalline sleeve, the compressive strength of the Ni-Fe coating material has been investigated at elevated temperatures. Figure 2 shows results from compression tests performed at room temperature on nanocrystalline Ni18wt.\%Fe cylindrical bulk samples after annealing the samples for $30 \mathrm{~min}$ at 423, 523, and $548 \mathrm{~K}$. As can be seen, the curves fall more or less on top of each other and resemble the one obtained for the as prepared sample, i.e. not annealed before being tested at room temperature. 
A tensile strength of $\sigma_{\mathrm{TS}}=2500 \mathrm{MPa}$ and an engineering strain of $\sim 14 \%$ is obtained. In case the compression tests are not performed at room temperature but at the respective temperature after annealing for $30 \mathrm{~min}$, the curves deviate from each other as seen in Fig. 3. The sample compressed at room temperature shows the highest yield/tensile strength and the lowest ductility when failure occurred ( 14\%). When the compression test was performed at elevated temperatures, a corresponding drop in strength and increase in ductility is observed. For example, when compressing the sample at $423 \mathrm{~K}$ after annealing, the fracture strain is with $\sim 30 \%$ already doubled. In fact, even higher fracture strains can be expected at higher testing temperatures. While failure occurred during tests performed at room temperature, $373 \mathrm{~K}$, and $423 \mathrm{~K}$, the compression tests at 473, 523 and $548 \mathrm{~K}$ were interrupted (the interrupted tests are shown with open symbols in Fig. 3). This means that even higher fracture stains than $30 \%$ would occur in these cases.

Characterization by use of TEM on transverse sections of the cylindrical samples revealed a coarser microstructure when the samples were compressed above room temperature. Figure 4 shows the microstructure of the samples compressed at room temperature, 423, 473 and 548 K. As can be seen, already at $423 \mathrm{~K}$ (Fig. 4(b)), the grains are slightly larger than at room temperature (Fig. 4(a)), while significant grain growth has occurred during compression at 473 and $548 \mathrm{~K}$ (Figs. 4(c and d)). Using transmission-EBSD on the respective TEM samples, the average grain size for the material compressed at 473 and $548 \mathrm{~K}$ was found to be $50 \pm 30$ $\mathrm{nm}$ and $100 \pm 50 \mathrm{~nm}$, and grains of more than $200 \mathrm{~nm}$ and $350 \mathrm{~nm}$, respectively, are present in the investigated areas. In Fig. 5, an EBSD orientation map of the sample compressed at $548 \mathrm{~K}$ is given in inverse pole figure colouring in growth direction. Grain boundaries are given as black lines and the obtained microstructure confirms the presence of sub-micrometer sized grains.

From differential scanning calorimetry measurements and EBSD studies of the same Ni-Fe electrodeposit it is known that the material is thermally stable up to $548 \mathrm{~K}$ [16], i.e. substantial grain growth was first observed at $573 \mathrm{~K}$. This is also confirmed by the TEM image given in Fig. 6, which shows the material annealed at $548 \mathrm{~K}$ and compressed at room temperature. A very fine-grained microstructure is visible in this case, i.e. no grain growth has occurred as in case of compression at $548 \mathrm{~K}$ (Fig. 4(d)). This means that deformation-induced grain growth takes place at temperatures way below the usual thermal stability of the nanomaterial. Dislocation theory can explain the substantial drop in yield strength/increase in engineering strain during compression at elevated temperatures. The larger grains allow strain hardening to occur by intra-granular dislocation accumulation [15] and this in turn leads to the larger fracture strains at higher temperatures. As described by Qin et al. [17] for bulk nanocrystalline Ni-19wt.\%Fe, grain boundary sliding sets first in at temperatures above 613 K.

\subsection{Micro-sandwich}

The micro-sandwich (Hybrix ${ }^{\mathrm{TM}}$, Lamera AB, Sweden) is thin and hollow, and consists of two steel face sheets separated by millions of polymer fibres ( $\sim 3 \mathrm{~mm}$ in length) that are attached by an adhesive [18]. The resulting advanced hybrid can be shaped like a metal sheet but the weight of the sandwich material is substantially reduced. To give the micro-sandwich further strength, a Nickel coating was pulse-electrodeposited on the face sheets. Figure 7 shows two EBSD orientation maps as obtained from Nickel coatings produced at different plating conditions. As can be seen, the grain size and texture of the coatings can vary substantially. While the microstructure in Fig. 7(a) is $<100>$ textured in growth direction and has a grain size of $365 \pm 219 \mathrm{~nm}$ with the largest grains being $1.7 \mu \mathrm{m}$, the EBSD orientation map in Fig. 7(b) has a random texture and a grain size of $81 \pm 77 \mathrm{~nm}$ (the largest grains measured were $650 \mathrm{~nm}$ ). Due to conservative data treatment (wild spikes are removed and a suitable 
orientation was only assigned to those zero solutions when the 6 nearest neighbours were equally indexed), a substantial fraction of pixels in the EBSD orientation map in Fig. 7(b) is not identified and shown in black. Hence, the grain size as obtained from the orientation map is slightly underestimated. Nano-indentation measurements were performed on the sample shown in Fig 7(a) and Vickers hardness values between 150 and $570 \mathrm{HV}$ were obtained depending on the location when using 1 and $5 \mathrm{mN}$, respectively (Fig. 8). The variation in hardness reflects the variation in grain size ranging from some nanometers to several micrometers. Hence, substantial improvements in hardness can be obtained when adjusting the plating parameters and tailoring the microstructure (e.g. by giving it a nanostructure like in Fig. 7(b)).

Additionally, it was also investigated whether it is possible to apply a coating on the polymer fibres of the sandwich structure. First, a thin Gold film was sputtered on the polymer fibres for making them conductive. Afterwards, a Nickel coating was pulse-electrodeposited on top. Figure 9 shows a side view of the sandwich structure (only one face sheet attached). Most of the fibres seen are coated by Nickel. However, there are also fibres visible that are still showing the Gold coating (reddish appearance). Hence, the Nickel layer is incomplete and further refinement of the coating process is required.

\section{Conclusions}

A strength increase is obtained when a nanocrystalline coating is applied as a sleeve on a PCM. However, when applying a load at elevated temperatures, the compression tests on the nanocrystalline sleeve material have shown that it is necessary to stabilize the nanomaterial against grain growth as a substantial drop in yield strength/increase in engineering strain has been observed. Instead of a solid solution like Ni-18wt.\%Fe, a supersaturated material (showing segregation) may be able to prevent deformation-induced grain growth to occur which otherwise compromises the initial strength increase.

Sandwich structures like the Hybrix ${ }^{\mathrm{TM}}$ by Lamera $\mathrm{AB}$ can be strengthened by applying a nano- or sub-microcrystalline coating on the face sheets. By controlling microstructure (e.g. grain size and texture) and thickness of the coating, the properties of the sandwich structure can be tailored. It is even possible to pulse-electroplate a nanocrystalline coating on the polymer fibres of the sandwich structure. However, further investigations are required to make sure that all fibres are coated during pulse-electrodeposition, i.e. that the conductive coating is continuous and no shading effects from neighbouring fibres occurs.

\section{Acknowledgements}

The authors are grateful to G.D. Hibbard, University of Toronto, Canada, for providing the PCMs and to Lamera AB, Sweden, for providing the Hybrix micro-sandwich. Thanks are also going to C. Godard, University of Kaiserslautern, Germany, for performing the nanoindentation tests. 


\section{References}

[1] M.F. Ashby, A.G. Evans, N.A. Fleck, L.J. Gibson, J.W. Hutchinson and H.N.G. Wadley: 'Metal Foams: A Design Guide', Butterworth-Heinemann, Boston, 2000.

[2] J. Banhart: 'Manufacture, characterisation and application of cellular metals and metal foams', Progress in Materials Science, 2001, 46, 559-632.

[3] B.A. Bouwhuis and G.D. Hibbard: 'Compression testing of periodic cellular sandwich cores’, Metallurgical and Metals Transactions B, 2006, 37B, 919-927.

[4] M.F. Ashby: 'Hybrids to fill holes in material property space', Philosophical Magazine, 2005, 85, 3235-3257.

[5] H.N.G. Wadley, N.A. Fleck, and A.G. Evans: 'Fabrication and structural performance of periodic cellular metal sandwich structures', Composites Science and Technology, 2003, 63, 2331-2343.

[6] E. Bele, B.A. Bouwhuis, and G.D. Hibbard: 'Work hardening as a strengthening mechanism in periodic cellular materials', Materials Science and Engineering A, 2008, 489, 29-37.

[7] B.A. Bouwhuis and G.D. Hibbard: 'Relative significance of in-situ work-hardening in deformation-formed micro-truss materials’, Materials Science and Engineering A, 2010, 527, 565-573.

[8] B.A. Bouwhuis, U. Klement and G.D. Hibbard: 'Microstructure in work-hardened microtruss materials given post-forming annealing treatments', Advances in Composite, Cellular and Natural Materials, Proceedings of 2010 TMS Annual Meeting, 2010.

[9] B.A. Bouwhuis, J.L. McCrea, G. Palumbo, and G.D. Hibbard: 'Mechanical properties of hybrid nanocrystalline metal foams', Acta Materialia, 2009, 57, 4046-4053.

[10] E. Bele, B.A. Bouwhuis, and G.D. Hibbard: 'Failure mechanisms in metal/metal nanocrystalline microtruss materials', Acta Materialia, 2009, 57, 5927-5935.

[11] P.W. Trimby, 'Orientation mapping of nanostructured materials using transmission Kikuchi diffraction in the scanning electron microscope', Ultramicroscopy, 2012, 120, 16-24.

[12] U. Klement, M. Shahabi-Navid, and G.D. Hibbard: 'Electrodeposited nanocrystalline NiFe with banded structure’, Materials Science Forum, 2012 706-709, 1618-1623.

[13] B.A. Bouwhuis, E. Bele, and G.D. Hibbard: 'Failure mechanisms during periodic cellular metal fabrication by perforation-stretching', Metallurgical and Materials Transactions A, 2008, 39A, 2329-3033.

[14] N. Mortazavi; MSc Thesis, Chalmers University of Technology, 2013.

[15] U. Klement, N. Mortazavi, J. Ahlström, C. Persson, and G.D. Hibbard: 'Mechanical properties of bulk- and hybrid nanocrystalline materials' New Frontiers of Nanomaterials, Proceedings of the $35^{\text {th }}$ Risø Int. Symposium on Materials Science, 2014, 349-355.

[16] U. Klement, M. Shahabi Navid, and G.D. Hibbard: 'Texture of electrodeposited nanocrystalline Ni-Fe with banded structure', Materials Science Forum, 2012, 702-703, 912-915.

[17] X.Y. Qin, X.G. Zhu, S. Gao, L.F. Chi, and J.S. Lee: 'Compression behaviour of bulk nanocrystalline Ni-Fe', Journal of Physics: Condensed Matter, 2002, 14, 2605-2620.

[18] http://www.lamera.se/eng/ (2015-07-09) 
Figures

Figure 1:
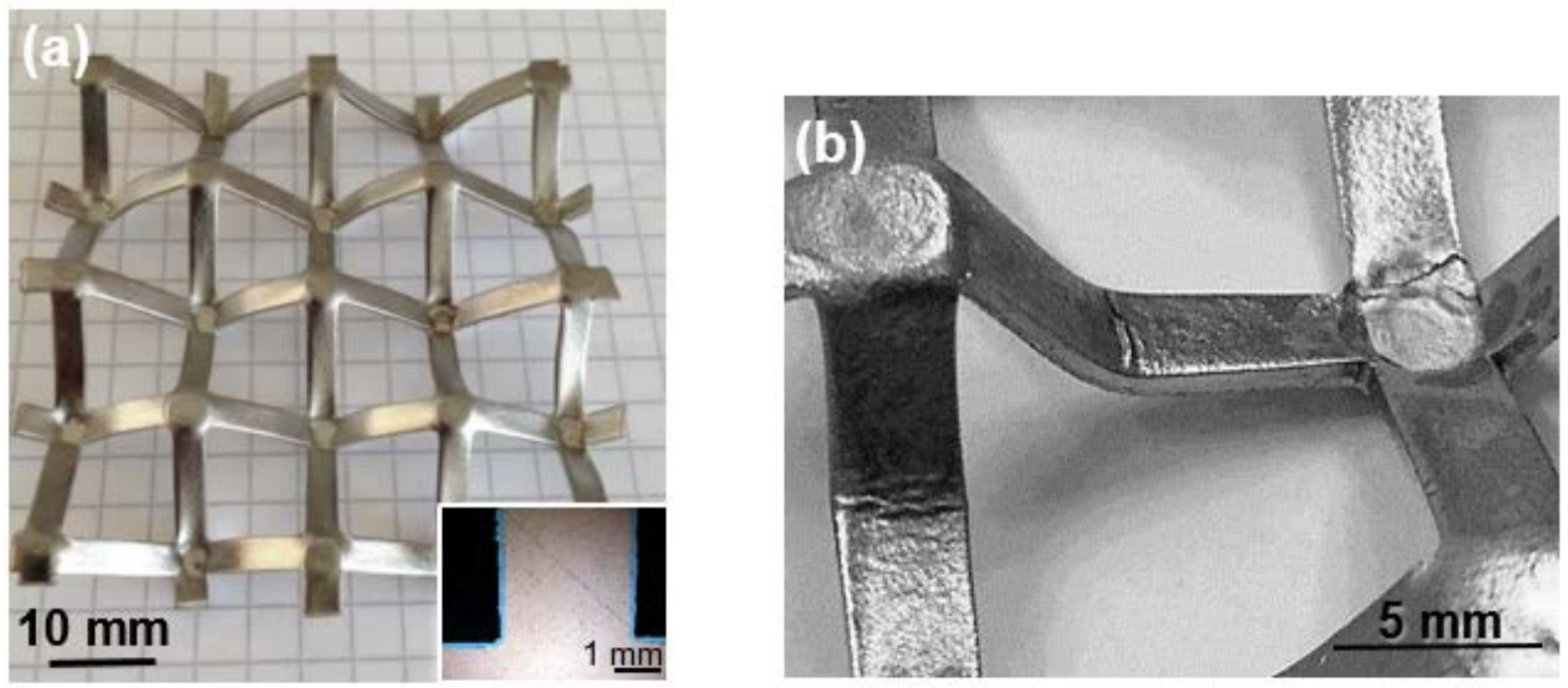

Figure 2:

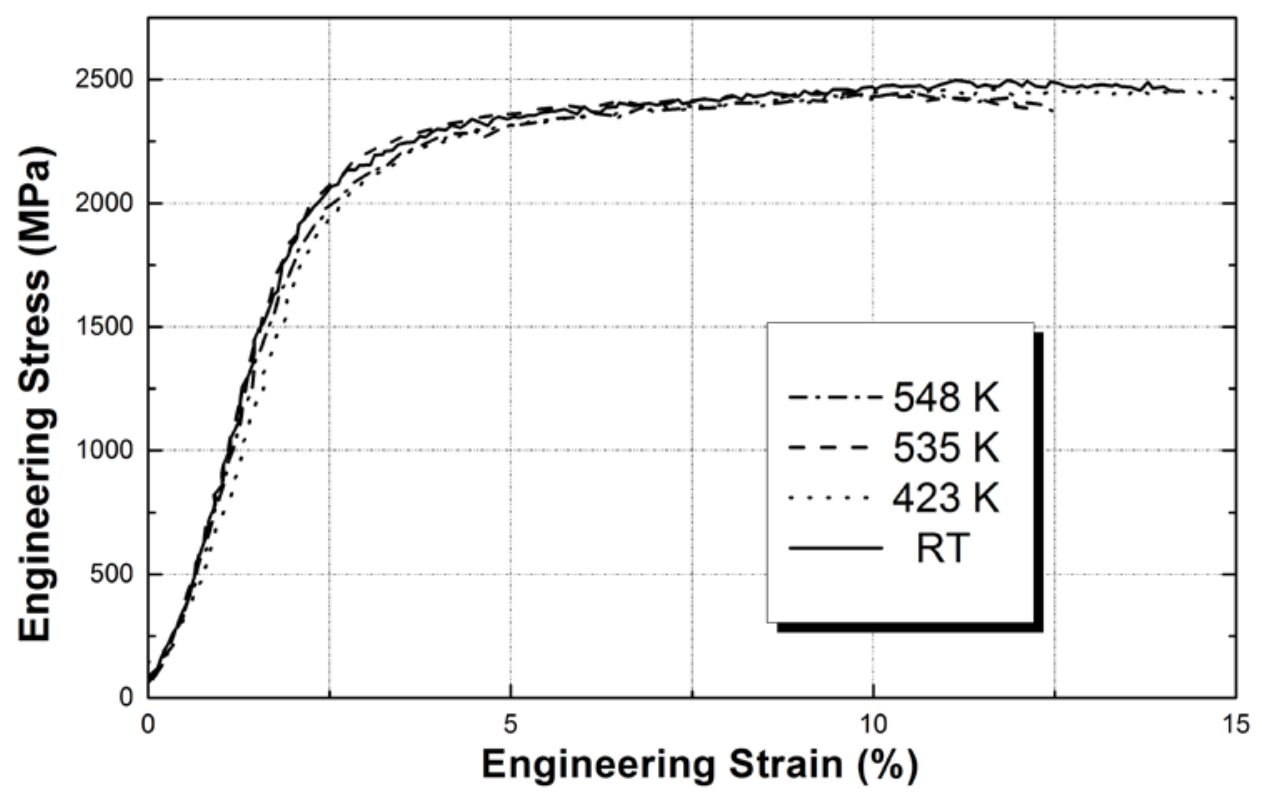


Figure 3:

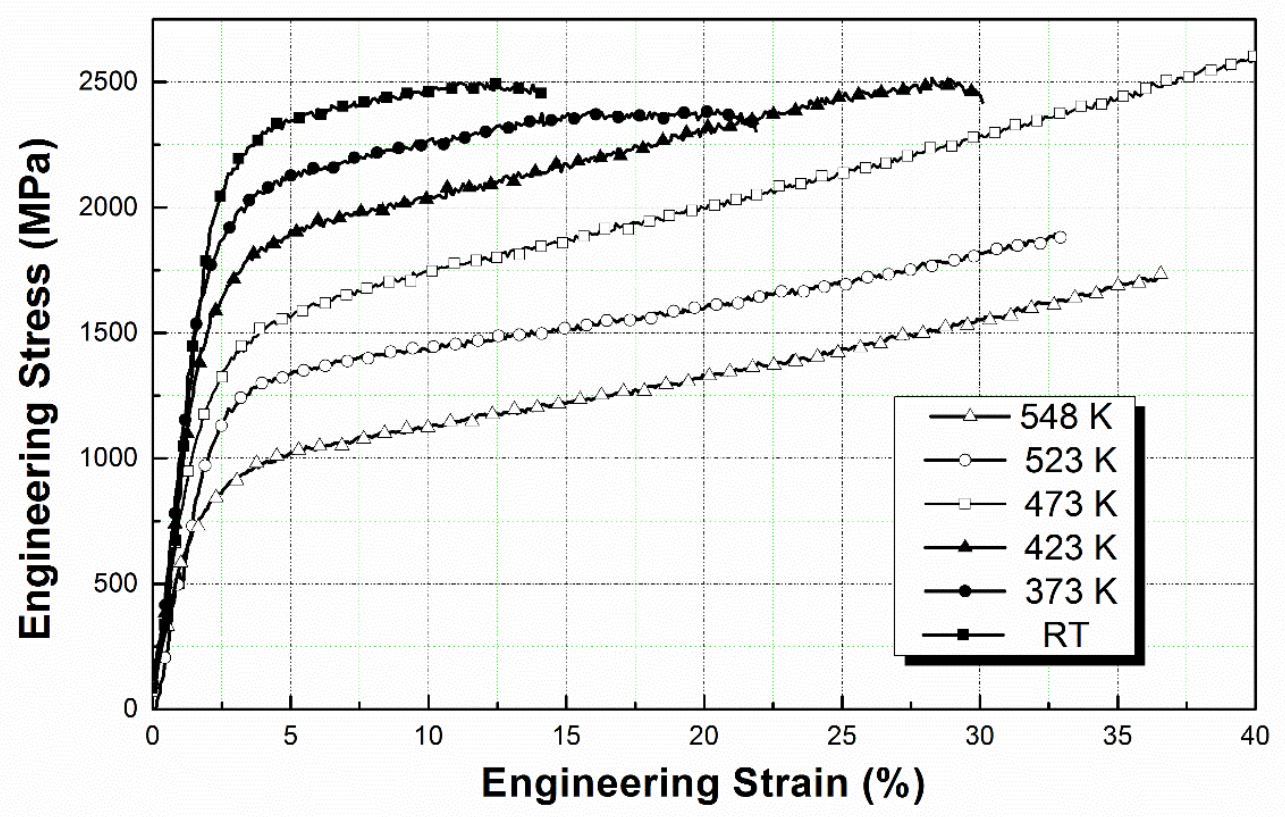

Figure 4:
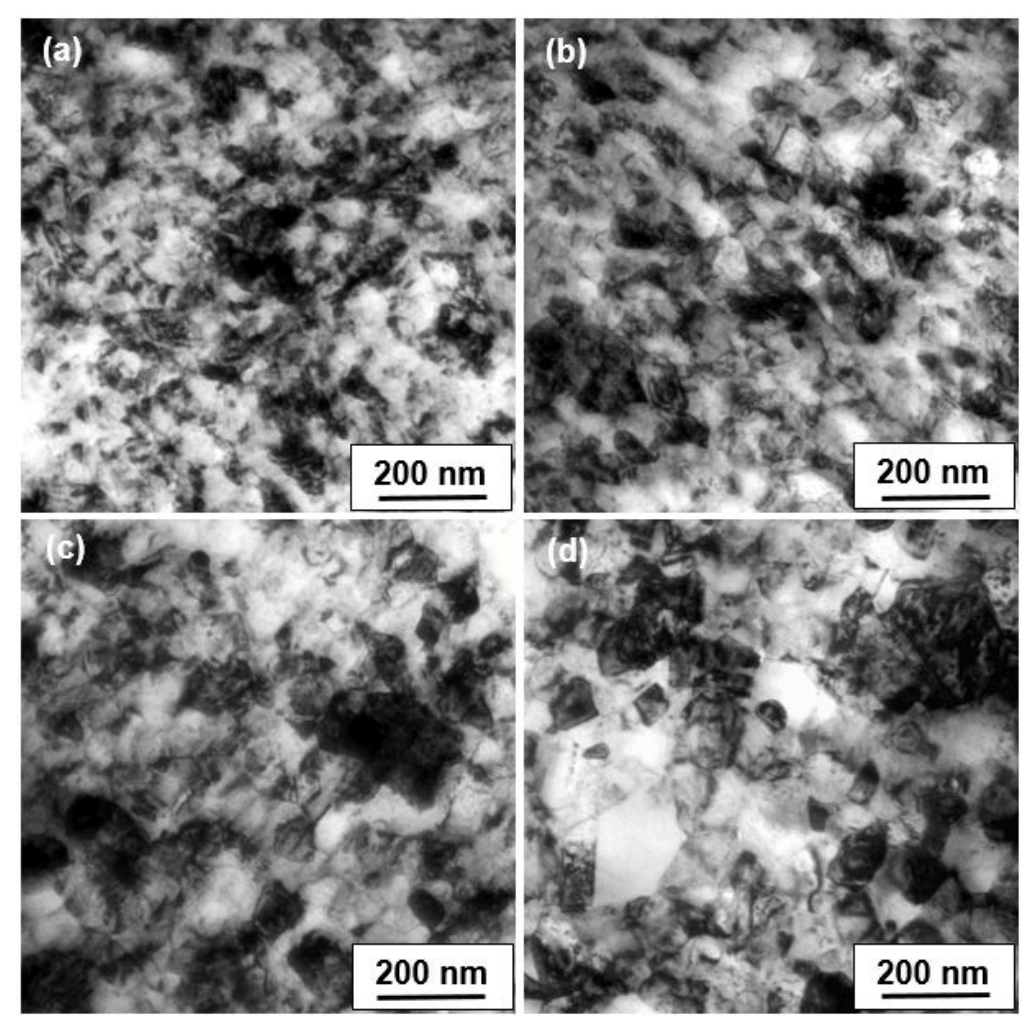
Figure 5:

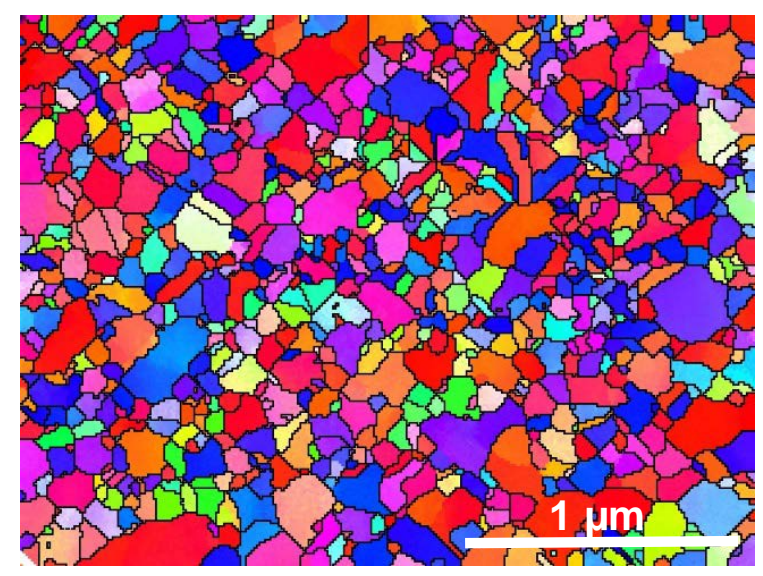

Figure 6:

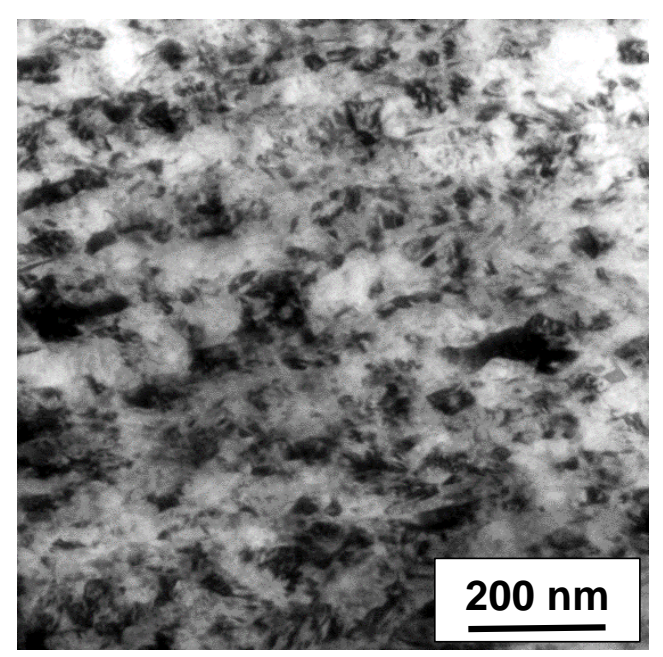

Figure 7:
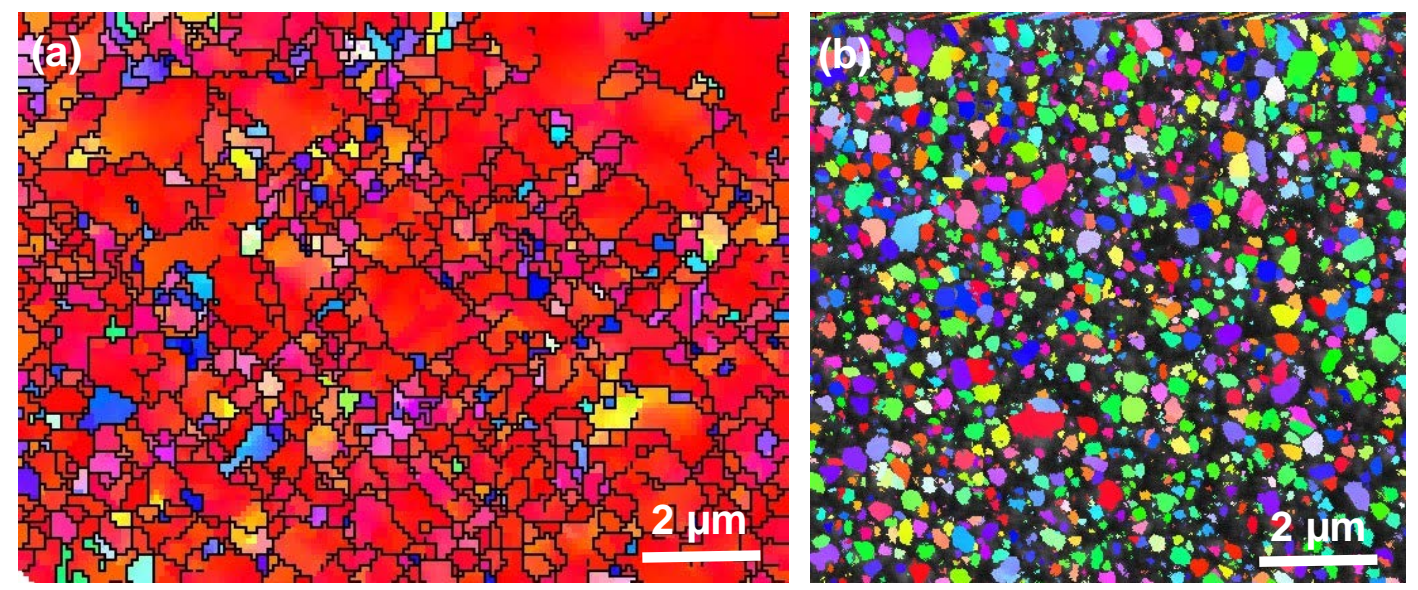
Figure 8:
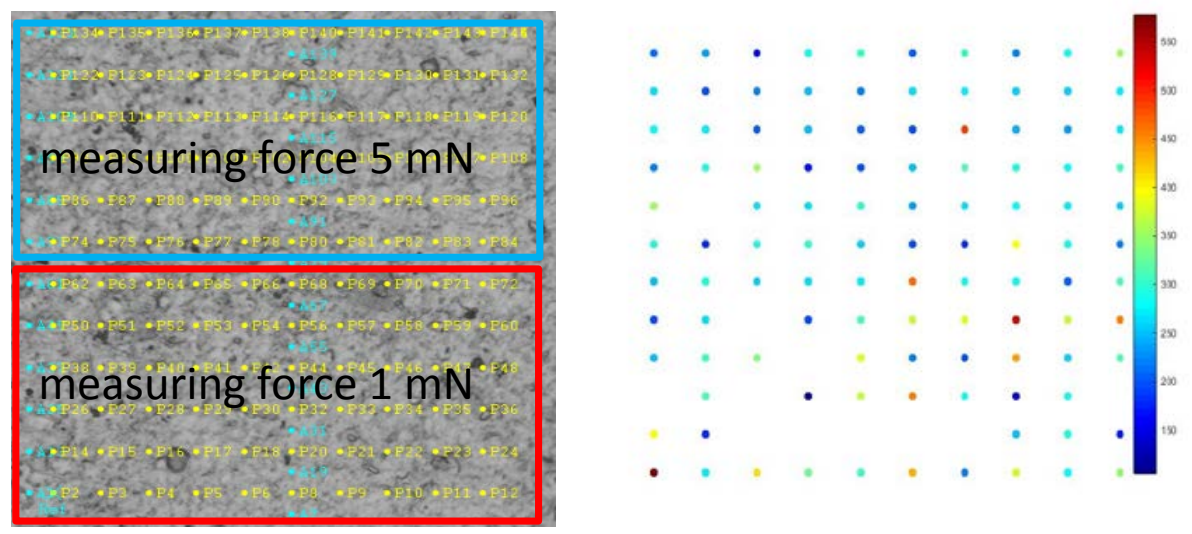

Figure 9:

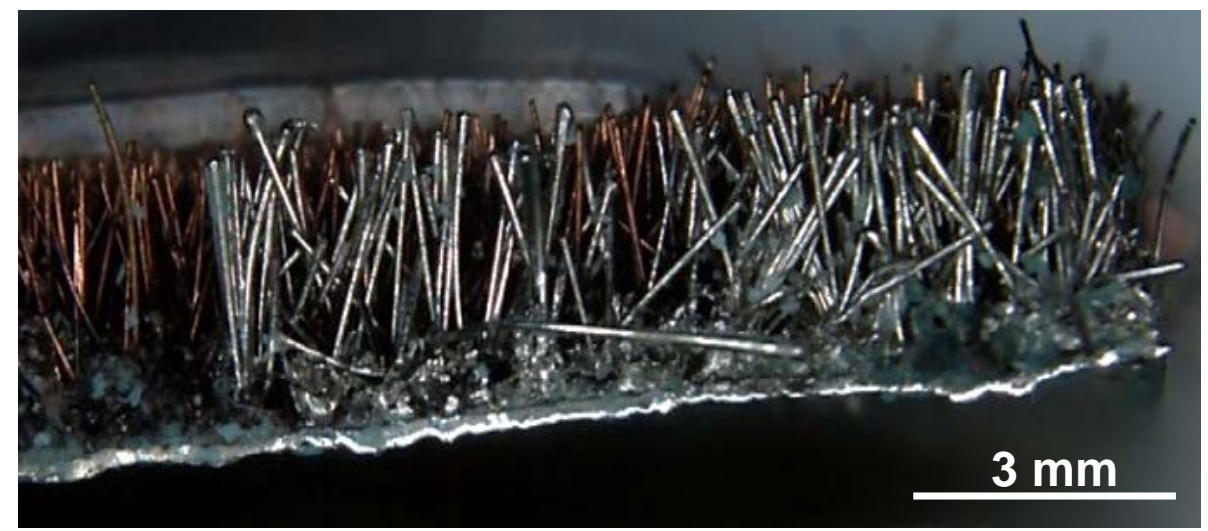




\section{Figure captions}

Figure 1: $\quad \mathrm{Cu} /$ nano Ni-Fe periodic cellular material (PCM): (a) Overview of 3D structure and inset showing the Cu-strut in cross section (top view) with the about $100 \mu \mathrm{m}$ thick coating; (b) Magnified image of a PCM after uniaxial free compression showing cracks at nodes and wrinkling at hinges.

Figure 2: Compressive engineering stress-strain curves of nanocrystalline Ni-Fe. The material was annealed for $30 \mathrm{~min}$ at the respective temperature before compression at room temperature.

Figure 3: Compressive engineering stress-strain curves of nanocrystalline Ni-Fe. The material was annealed for $30 \mathrm{~min}$ and afterwards compressed at the respective temperature. Open symbols illustrate that the compression test was interrupted before failure occurred.

Figure 4: TEM images of (a) as prepared Ni-Fe, and samples compressed at (b) $423 \mathrm{~K}$, (c) $473 \mathrm{~K}$, and (d) $548 \mathrm{~K}$.

Figure 5: EBSD orientation map of pulse-electrodeposited Ni-Fe compressed at $548 \mathrm{~K}$ after an annealing treatment for $30 \mathrm{~min}$. Sub-micrometer sized grains are present in the microstructure.

Figure 6: TEM images of Ni-Fe sample annealed for $30 \mathrm{~min}$ at $548 \mathrm{~K}$ and compressed at room temperature.

Figure 7: EBSD orientation maps as obtained from Nickel coatings on the steel face sheets of the micro-sandwich produced at different plating conditions. (a) $<100>$ texture and microcrystalline microstructure; (b) random texture and nanocrystalline microstructure.

Figure 8: Nano-indentation measurements obtained on the Nickel coating pulse-plated on stainless steel shown in Fig. 7(a). The hardness values vary locally between 150 and $570 \mathrm{HV}$.

Figure 9: Side view of the micro-sandwich with only one face sheet attached. The polymer fibres were first sputtered with gold, afterwards a Nickel coating was pulse electrodeposited. 